BMJ Open

Diabetes

Research

\& Care

\section{Risk of hypertension in middle-aged and elderly participants with newly diagnosed type 2 diabetes and prediabetes}

To cite: Sasaki N, Ozono R, Maeda R, et al. Risk of hypertension in middle-aged and elderly participants with newly diagnosed type 2 diabetes and prediabetes. BMJ Open Diab Res Care 2020;8:e001500. doi:10.1136/ bmjdrc-2020-001500

- Additional material is published online only. To view, please visit the journal online (http://dx.doi.org/10.1136/ bmjdrc-2020-001500).

Received 24 April 2020 Revised 2 July 2020 Accepted 30 July 2020

A Check for updates

C Author(s) (or their employer(s)) 2020. Re-use permitted under CC BY-NC. No commercial re-use. See rights and permissions. Published by BMJ.

${ }^{1}$ Health Management and Promotion Center, Hiroshima Atomic Bomb Casualty Council, Hiroshima, Japan

${ }^{2}$ Department of General Medicine, Hiroshima University Graduate School of Biomedical and Health Sciences,

Hiroshima, Japan

${ }^{3}$ Department of Cardiovascular Regeneration and Medicine, Research Institute for Radiation Biology and Medicine, Hiroshima University Graduate School of Biomedical and Health Sciences, Hiroshima, Japan

Correspondence to Dr Nobuo Sasaki; nb7ssk7@sd6.so-net.ne.jp

\section{ABSTRACT}

Introduction Little is known about the risk of hypertension in patients with the early stage of type 2 diabetes. We investigated the risk of hypertension in participants with newly diagnosed type 2 diabetes and prediabetes.

Research design and methods This is a retrospective cohort study consisting of 2136 middle-aged participants (1022 with normal fasting glucose/normal glucose tolerance (NFG/NGT), 418 with impaired fasting glucose (IFG), 466 with impaired glucose tolerance (IGT) and 230 with diabetes) and 3426 elderly participants (1762 with NFG/NGT, 599 with IFG, 781 with IGT, and 284 with diabetes). All participants underwent $75 \mathrm{~g}$ oral glucose tolerance tests at baseline.

Results Over a median 59-month follow-up period, 459 middle-aged and 1170 elderly participants developed hypertension. In middle-aged participants, the odds of incident hypertension were significantly higher in those with IFG (OR 1.40; $p=0.019)$, IGT (OR 1.49; $p=0.004)$, and diabetes (OR 1.55; $p=0.013$ ) than those with NFG/ NGT, which was no longer significant after adjustment for body mass index. Subgroup analysis showed that the risk of hypertension was significantly higher in diabetes than NFG/NGT only in participants without obesity. Conversely, obesity was a risk factor of hypertension only in those with IFG and NFG/NGT. In elderly participants, there was no difference in the risk of hypertension among the NFG/NGT, IFG, IGT and diabetes groups.

Conclusions The risk of hypertension is modest in participants with newly diagnosed type 2 diabetes and prediabetes. Our findings suggest that the early stages of type 2 diabetes and prediabetes may be a key period for reducing hypertension, given the pronounced risk of hypertension in patients with diabetes reported in previous studies. In terms of reducing the risk for hypertension, obesity treatment might be advantageous in the early stages rather than the advanced stages of impaired glucose metabolism.

\section{INTRODUCTION}

Hypertension and type 2 diabetes frequently coexist, and these two conditions substantially overlap in etiology and underlying mechanisms. ${ }^{1}$ Patients with type 2 diabetes usually exhibit insulin resistance, ${ }^{2}$ which is

\section{Significance of this study}

What is already known about this subject?

> Several studies have reported the marked risk of hypertension in middle-aged patients with type 2 diabetes.

- Newly diagnosed diabetes has clinical features distinct from long-standing diabetes.

What are the new findings?

- The relationship between hypertension and type 2 diabetes was modestly significant in middle-aged participants with newly diagnosed type 2 diabetes and those with prediabetes, which was no longer significant after adjustment for body mass index.

- In the elderly population, there was no difference in the risk of hypertension among those with normal fasting glucose/normal glucose tolerance (NFG/ NGT), prediabetes and diabetes.

- Obesity is associated with the development of hypertension only in participants with impaired fasting glucose and those with NFG/NGT.

How might these results change the focus of research or clinical practice?

- Our findings suggest that the early stages of type 2 diabetes and prediabetes may be a key period for reducing hypertension, given the pronounced risk of hypertension in patients with diabetes reported in previous studies.

- In terms of reducing the risk for hypertension, obesity treatment might be advantageous in the early stages rather than the advanced stages of impaired glucose metabolism.

a contributing factor to the development of hypertension. ${ }^{3}{ }^{4}$ Several studies have shown a pronounced risk of hypertension in patients with type 2 diabetes. ${ }^{5-8}$ Conversely, other studies have reported no significant association between plasma glucose level and the incidence of hypertension. ${ }^{9-11}$ A recent Mendelian randomization analysis showed only a slight increased risk of hypertension in genetically linked type 2 diabetes. ${ }^{12}$ 
These lines of evidence evoke the possibility that the pronounced risk of hypertension in patients with type 2 diabetes is not mainly due to hyperglycemia per se but to confounding factors, which were not adequately taken in account. ${ }^{13}$ In previous observational studies, the group of patients with type 2 diabetes was composed of participants with newly diagnosed diabetes and patients being actively treated for diabetes. ${ }^{5-8}$ Arterial stiffness, vascular damage, and subclinical renal dysfunction usually develop during the natural course of type 2 diabetes, ${ }^{14-16}$ and all of these factors could predispose the patient to the development of hypertension. ${ }^{17} 18$ Therefore, newly diagnosed type 2 diabetes is considered to have distinct clinical features characterized by shorter duration of hyperglycemic exposure as compared with long-standing type 2 diabetes. To our best knowledge, however, there is no study to test whether newly diagnosed type 2 diabetes carries an increased risk of hypertension as compared with normal fasting glucose/normal glucose tolerance (NFG/NGT). In addition, it remains unclear whether prediabetes including impaired fasting glucose (IFG), and impaired glucose tolerance (IGT) per se elicits hypertension. ${ }^{5619-21}$ Furthermore, little is known about the risk of hypertension in elderly patients with type 2 diabetes or prediabetes, although its prevalence continues to increase with the growing elderly population. ${ }^{22}$

In this retrospective cohort study, we investigated the risk of hypertension in a large-scale population who had no history of treatment for diabetes. All participants were classified as having NFG/NGT, IFG, IGT, and diabetes using the $75 \mathrm{~g}$ oral glucose tolerance tests (OGTT). To clarify the age-related difference in the risk of hypertension, we divided the participants into middle-aged and elderly groups. In addition, we assessed the impact of obesity on the incidence of hypertension in participants with different stages of impaired glucose metabolism.

\section{METHODS}

\section{Study population}

This study used data collected from the Hiroshima Study on Glucose Metabolism and Cardiovascular Diseases (Hiroshima GMCVD), which was a cross-sectional and longitudinal study that examined the inter-relationship among impaired glucose metabolism, hypertension, and cardiovascular disease (CVD). Participants were recruited from examinee of general health check-ups provided as a governmental service for atomic bomb survivors in Hiroshima City at the Health Management and Promotion Center of Hiroshima Atomic Bomb Casualty Council, on the basis of the following criteria: (1) they had no history of treatment for diabetes, (2) they had suspicious impaired glucose metabolism based on the screening of fasting plasma glucose (FPG) more than $5.6 \mathrm{mmol} / \mathrm{L}$, postprandial 1-hour plasma glucose more than $8.6 \mathrm{mmol} / \mathrm{L}$, postprandial 2-hour plasma glucose more than $7.8 \mathrm{mmol} / \mathrm{L}$ or postprandial 3-hour plasma glucose more than $6.7 \mathrm{mmol} / \mathrm{L}$, and (3) they agreed to

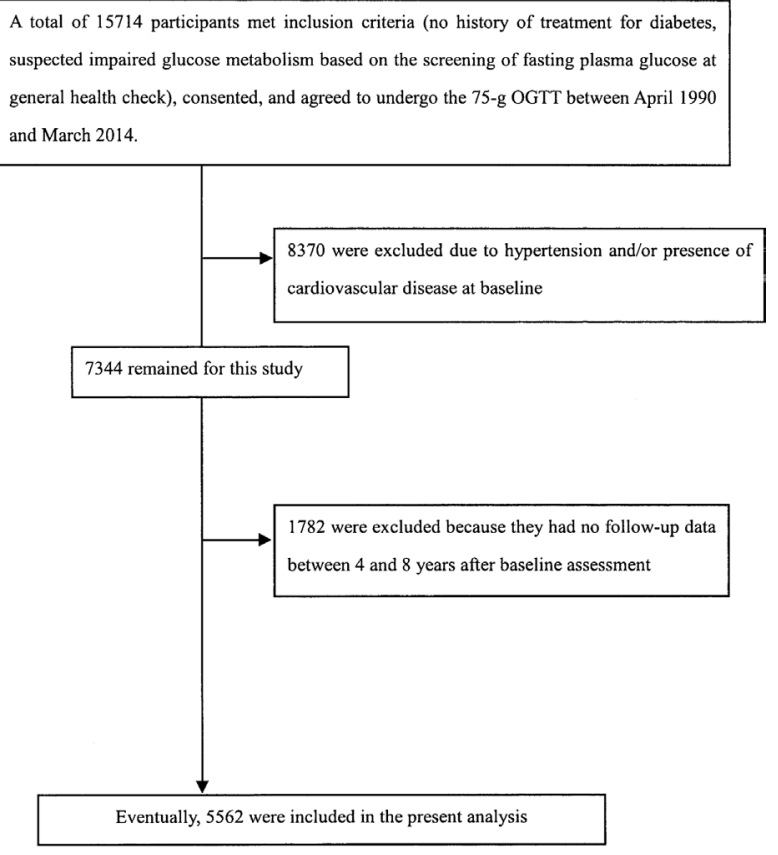

Figure 1 Flow chart for the selection of participants. OGTT, oral glucose tolerance tests.

undergo the $75 \mathrm{~g}$ OGTT. We obtained informed consent from all participants during their health examinations. We asked all participants about their regular medications and medical histories, including treatment for hypertension, dyslipidemia, and CVD, as well as their drinking and smoking habits.

Of the 15714 participants who underwent $75 \mathrm{~g}$ OGTT between April 1990 and March 2014 from the Hiroshima GMCVD database, 8370 were excluded due to hypertension (defined as taking antihypertensive medications, and/or having systolic blood pressure $\geq 140 \mathrm{~mm} \mathrm{Hg}$, and/ or diastolic blood pressure $\geq 90 \mathrm{~mm} \mathrm{Hg})^{23}$ and/or having a history of CVD at baseline. Of the remaining normotensive 7344 participants, 1782 were excluded due to lack of follow-up data between 4 and 8 years after baseline health examinations (figure 1). This study was registered under the University Hospital Medical Information Network protocol registration system (ID: UMIN000036648).

\section{Covariate and outcome definition}

Incidence of hypertension was defined as taking antihypertensive medications and/or having systolic blood pressure $\geq 140 \mathrm{~mm} \mathrm{Hg}$ and/or diastolic blood pressure $\geq 90 \mathrm{~mm} \mathrm{Hg}^{23}$ on the occasion of medical follow-up. As for participants' personal habits, current smoker was defined as having a current smoking habit regardless of the number of cigarettes smoked per day, and habitual drinker was defined as drinking alcohol $\geq 20 \mathrm{~g}$ per day.

\section{Measurements}

The $75 \mathrm{~g}$ OGTT was conducted in the morning after an overnight fast. For measurements of plasma glucose, samples were drawn just before and 30,60, and $120 \mathrm{~min}$ after ingestion of glucose. Plasma glucose was measured 
using the hexokinase/glucose-6-phosphate dehydrogenase method at our institution in all participants. We classified the participants into four groups according to their glycemic status as defined by American Diabetes Association criteria ${ }^{24}$ : (1) NFG)/NGT, defined as FPG $<5.6 \mathrm{mmol} / \mathrm{L}$ and 2-hour postload glucose $<7.8 \mathrm{mmol} / \mathrm{L}$; (2) IFG, defined as FPG $5.6-6.9 \mathrm{mmol} / \mathrm{L}$ and 2-hour postload glucose $<7.8 \mathrm{mmol} / \mathrm{L}$; (3) IGT, defined as FPG $<7.0 \mathrm{mmol} / \mathrm{L}$ and 2-hour postload glucose $7.8-11.0 \mathrm{mmol} / \mathrm{L}$; (4) diabetes, defined as FPG $\geq 7.0 \mathrm{mmol} / \mathrm{L}$ and/or 2-hour postload glucose $\geq 11.1 \mathrm{mmol} / \mathrm{L}$.

Serum immunoreactive insulin (IRI) concentrations were measured using a radioimmunoassay at the SRL Laboratories (SRL Inc, Tokyo, Japan) between April 1990 and March 2003. We assessed insulin resistance using the following two parameters:

1. Homeostasis model assessment of insulin resistance (HOMA-IR), which was calculated as insulin (mI$\mathrm{U} / \mathrm{L}) \times$ glucose $(\mathrm{mg} / \mathrm{dL}) / 405 .{ }^{25}$

2. The Matsuda index as a measure for whole-body insulin resistance, which was calculated as 10000 divided by the square root of FPG $\times$ fasting IRI $\times 2$-hour postload glucose $\times 2$-hour postload IRI $^{26} 27$ in middle-aged participants between April 1990 and March 2003.

Blood pressure was measured in subjects seated in a chair with back support and their arm supported at heart level after a rest period of $>5 \mathrm{~min}$ at the time of their health examinations. For blood pressure measurements, we used a mercury sphygmomanometer before 2012 and a digital automatic blood pressure measuring instrument (Terumo (Tokyo, Japan) or Omron Healthcare (Kyoto, Japan)) after 2013. The estimated glomerular filtration rate (eGFR) from creatinine levels was calculated as follows: eGFRcreat $\left(\mathrm{mL} / \mathrm{min} / 1.73 \mathrm{~m}^{2}\right)=194 \times \mathrm{Cr}^{-1.094} \times$ age $^{-0.287} \times 0.739$ (if female). ${ }^{28}$ Urine protein levels were semiquantified using urine-protein-reagent testing strip. Results of the urine protein level were recorded as $(-)$, trace, $(1+),(2+)$, or $(3+)$. The presence of proteinuria was defined as $(1+)$ or over.

\section{Participant classification}

We divided the participants into a middle-aged group defined as age $<65$ years $(n=2136)$ and an elderly group defined as age $\geq 65$ years $(n=3426)$. We defined obesity as body mass index (BMI) $\geq 25 \mathrm{~kg} / \mathrm{m}^{229}$ and used this to subdivide the middle-aged group of patients into an obesity group $(n=447)$ and a non-obesity group $(n=1689)$.

\section{Statistical analysis}

Continuous variables are expressed as mean $\pm \mathrm{SD}$, and normality of continuous variables was examined with the Kolmogorov-Smirnov test. Differences among the four groups (ie, NFG/NGT, IFG, IGT, and diabetes) were analyzed with the Kruskal-Wallis test. Categorical variables were summarized as percentages and were analyzed using the $\chi^{2}$ test. The Cochran-Armitage trend test was used to examine the significance of trends in the proportion of incident hypertension in the order of participants with NFG/NGT, with IFG, with IGT, and with diabetes. We evaluate the unadjusted and adjusted ORs of incident hypertension based on the baseline categories of impaired glucose metabolism (NFG/NGT, IFG, IGT, and diabetes) using univariate and multivariate logistic regression analyses with the following three models: model 1 included age, and sex; model 2 additionally included follow-up period, eGFR, serum total cholesterol level, smoking (never smoker, current smoker or former smoker), and habitual drinker (yes or no) in model 1; model 3 additionally included BMI in model 2. Next, we stratified the participants with obesity and those without obesity in the middle-aged population and evaluate the unadjusted and adjusted ORs of incident hypertension based on the baseline categories of impaired glucose metabolism using univariate and multivariate logistic regression analyses with model 3 . We estimated the unadjusted and adjusted ORs of obesity for incident hypertension in each of the baseline impaired glucose metabolism categories among middle-aged participants using univariate and multivariate logistic regression analyses with the model including age, gender, follow-up period, eGFR, serum total cholesterol level, smoker, and habitual drinker. In participants with available urine protein of date, we performed additional analysis to estimate the unadjusted and adjusted ORs of both renal dysfunction (defined as eGFR $<60 \mathrm{~mL} / \mathrm{min} / 1.73 \mathrm{~m}^{2}$ ) and proteinuria for incident hypertension in both middle-aged $(n=2130)$ and elderly groups $(n=3391)$. The multivariate model included age, gender, BMI, follow-up period, serum total cholesterol level, smoking, alcohol consumption, and impaired glucose metabolism states (NFG/NGT, IFG, IGT, and diabetes). We considered $\mathrm{p}<0.05$ as statistically significant. All statistical analyses were performed using the JMP V.14.2 statistical software.

\section{RESULTS}

A total of 5562 participants (2398 men and 3164 women; mean age: $66.2 \pm 6.8$ years; mean BMI: $22.7 \pm 3.0 \mathrm{~kg} / \mathrm{m}^{2}$ ) were included in the study. Of those, $803(14 \%)$ were current smokers, 834 (15\%) were former smokers, 3925 $(71 \%)$ were non-smokers who had never smoked, and $965(17 \%)$ were habitual drinkers. Table 1 shows clinical characteristics and results of OGTT in the middleaged and elderly participants with NFG/NGT, IFG, IGT, and diabetes. BMI increased in the order of NFG/NGT, IFG, IGT, and diabetes in both middle-aged and elderly participants.

Over a median 59-month follow-up period, 179 participants $(17.5 \%$ of 1022$)$ with NFG/NGT, $100(23.9 \%$ of 418) with IFG, 119 (25.5\% of 466) with IGT, and 61 (26.5\% of 230) with diabetes developed hypertension in the middle-aged population, whereas 571 (32.4\% of 1762) with NFG/NGT, 222 (37.1\% of 599) with IFG, 278 (35.6\% of 781$)$ with IGT, and 99 (34.9\% of 284) with diabetes developed hypertension in the elderly 
Table 1 Baseline characteristics of participants with different stages of impaired glucose metabolism

\begin{tabular}{|c|c|c|c|c|c|}
\hline & NGT/NFG & IFG & IGT & Diabetes & $P$ value \\
\hline \multicolumn{6}{|l|}{ Middle-aged group $(n=2136)$} \\
\hline $\mathrm{N}$ & 1022 & 418 & 466 & 230 & \\
\hline Mean age, years & $59.4 \pm 4.0$ & $59.3 \pm 3.8$ & $59.4 \pm 3.9$ & $58.5 \pm 4.2$ & 0.020 \\
\hline Female, n (\%) & $623(61)$ & $191(46)$ & $225(48)$ & $100(43)$ & $<0.001$ \\
\hline BMI, kg/m² & $22.0 \pm 2.6$ & $23.1 \pm 2.8$ & $23.6 \pm 2.9$ & $24.2 \pm 3.4$ & $<0.001$ \\
\hline \multicolumn{6}{|l|}{ Smoking } \\
\hline Never smoker, n (\%) & $733(72)$ & $266(64)$ & $306(66)$ & $134(58)$ & $<0.001$ \\
\hline Current smoker, n (\%) & $181(18)$ & $80(19)$ & $103(22)$ & $68(30)$ & \\
\hline Former smoker, n (\%) & $108(11)$ & $72(17)$ & $57(12)$ & $28(12)$ & \\
\hline Habitual drinker, n (\%) & $152(15)$ & $102(24)$ & $114(24)$ & $51(22)$ & $<0.001$ \\
\hline $\mathrm{SBP}, \mathrm{mm} \mathrm{Hg}$ & $119 \pm 11$ & $122 \pm 11$ & $122 \pm 10$ & $122 \pm 10$ & $<0.001$ \\
\hline DBP, $\mathrm{mm} \mathrm{Hg}$ & $72 \pm 8$ & $74 \pm 7$ & $74 \pm 8$ & $74 \pm 8$ & $<0.001$ \\
\hline Fasting $\mathrm{PG}, \mathrm{mmol} / \mathrm{L}$ & $5.1 \pm 0.3$ & $5.9 \pm 0.3$ & $5.7 \pm 0.6$ & $7.4 \pm 1.6$ & $<0.001$ \\
\hline 2-hour PG after ingestion of glucose, $\mathrm{mmol} / \mathrm{L}$ & $5.8 \pm 1.1$ & $6.2 \pm 1.0$ & $8.9 \pm 0.9$ & $14.2 \pm 4.0$ & $<0.001$ \\
\hline Antihyperlipidemic medication, $\mathrm{n}(\%)$ & $156(15)$ & $77(18)$ & $82(18)$ & $36(16)$ & 0.431 \\
\hline Total cholesterol, $\mathrm{mmol} / \mathrm{L}$ & $5.7 \pm 0.9$ & $5.7 \pm 0.9$ & $5.8 \pm 0.9$ & $5.8 \pm 0.9$ & 0.015 \\
\hline eGFR, $\mathrm{mL} / \mathrm{min} / 1.73 \mathrm{~m}^{2}$ & $78.9 \pm 19.8$ & $78.2 \pm 18.9$ & $80.2 \pm 22.6$ & $83.7 \pm 21.4$ & 0.003 \\
\hline Follow-up period, months & $60.6 \pm 7.5$ & $61.1 \pm 8.2$ & $61.8 \pm 8.5$ & $62.2 \pm 8.0$ & 0.004 \\
\hline \multicolumn{6}{|l|}{ Elderly group $(\mathrm{n}=3426)$} \\
\hline $\mathrm{N}$ & 1762 & 599 & 781 & 284 & \\
\hline Mean age, years & $70.5 \pm 4.1$ & $70.6 \pm 4.1$ & $70.4 \pm 4.1$ & $70.2 \pm 4.2$ & 0.515 \\
\hline Female, n (\%) & $1134(64)$ & $335(56)$ & $418(54)$ & $138(49)$ & $<0.001$ \\
\hline $\mathrm{BMI}, \mathrm{kg} / \mathrm{m}^{2}$ & $22.1 \pm 2.8$ & $23.0 \pm 3.0$ & $23.3 \pm 3.1$ & $23.6 \pm 3.2$ & $<0.001$ \\
\hline \multicolumn{6}{|l|}{ Smoking, n (\%) } \\
\hline Never smoker & $1336(76)$ & $433(72)$ & $530(68)$ & $187(66)$ & $<0.001$ \\
\hline Current smoker & $166(9)$ & $53(9)$ & $102(13)$ & $50(18)$ & \\
\hline Former smoker & $260(15)$ & $113(19)$ & $149(19)$ & $47(17)$ & \\
\hline Habitual drinker & $233(13)$ & $110(18)$ & $137(18)$ & $66(23)$ & $<0.001$ \\
\hline $\mathrm{SBP}, \mathrm{mm} \mathrm{Hg}$ & $121 \pm 11$ & $124 \pm 10$ & $123 \pm 10$ & $123 \pm 10$ & $<0.001$ \\
\hline DBP, $\mathrm{mm} \mathrm{Hg}$ & $71 \pm 8$ & $73 \pm 7$ & $72 \pm 8$ & $72 \pm 8$ & $<0.001$ \\
\hline Fasting $P G, \mathrm{mmol} / \mathrm{L}$ & $5.1 \pm 0.3$ & $5.8 \pm 0.3$ & $5.6 \pm 0.5$ & $6.9 \pm 1.3$ & $<0.001$ \\
\hline $\begin{array}{l}\text { 2-hour PG after ingestion of glucose, } \\
\mathrm{mmol} / \mathrm{L}\end{array}$ & $5.9 \pm 1.1$ & $6.2 \pm 1.1$ & $8.9 \pm 0.9$ & $13.4 \pm 3.3$ & $<0.001$ \\
\hline Antihyperlipidemic medication, $\mathrm{n}(\%)$ & $364(21)$ & $116(19)$ & $170(22)$ & $57(20)$ & 0.739 \\
\hline Total cholesterol ${ }^{\star}, \mathrm{mmol} / \mathrm{L}$ & $5.6 \pm 0.9$ & $5.7 \pm 0.9$ & $5.6 \pm 0.9$ & $5.7 \pm 1.0$ & 0.248 \\
\hline eGFR $†, \mathrm{~mL} / \mathrm{min} / 1.73 \mathrm{~m}^{2}$ & $70.6 \pm 14.6$ & $70.6 \pm 17.7$ & $72.9 \pm 19.3$ & $73.2 \pm 18.8$ & 0.070 \\
\hline Follow-up period, months & $60.3 \pm 6.9$ & $60.4 \pm 7.4$ & $60.3 \pm 7.1$ & $61.3 \pm 7.6$ & 0.179 \\
\hline
\end{tabular}

Data are expressed as mean \pm SD or as number and percentage.

*3425 subjects.

†426 subjects because of missing values.

BMI, body mass index; DBP, diastolic blood pressure; eGFR, estimated glomerular filtration rate; IFG, impaired fasting glucose; IGT, impaired glucose tolerance; NFG, normal fasting glucose; NGT, normal glucose tolerance; PG, plasma glucose; SBP, systolic blood pressure.

population. The rate of incident hypertension significantly increased in the order of NFG/NGT, IFG, IGT, and diabetes in the middle-aged population ( $\mathrm{p}$ for trend $<0.001$ ), whereas no significant differences were seen in the elderly population.
Table 2 shows the unadjusted and adjusted ORs for the incidence of hypertension in the categories of impaired glucose metabolism. In the middle-aged group, the risk of hypertension was significantly associated with the presence of IFG (OR 1.40; 95\% CI 1.06 to 1.86; $\mathrm{p}=0.019$ ), IGT 
Table 2 Univariate and multivariate ORs for incident hypertension

\begin{tabular}{|c|c|c|c|c|c|c|c|c|c|c|c|c|c|}
\hline \multirow[b]{2}{*}{ Variables } & \multirow[b]{2}{*}{$\mathbf{N}$} & \multicolumn{3}{|c|}{ Univariate } & \multicolumn{3}{|c|}{ Model 1} & \multicolumn{3}{|c|}{ Model 2} & \multicolumn{3}{|c|}{ Model 3} \\
\hline & & OR & $95 \% \mathrm{Cl}$ & $P$ value & OR & $95 \% \mathrm{Cl}$ & $P$ value & OR & $95 \% \mathrm{Cl}$ & $P$ value & OR & $95 \% \mathrm{Cl}$ & $P$ value \\
\hline \multicolumn{14}{|c|}{ Middle-aged group } \\
\hline NFG/NGT & 1022 & 1 & & & 1 & & & 1 & & & 1 & & \\
\hline IFG & 418 & 1.48 & (1.12 to 1.95$)$ & 0.005 & 1.40 & (1.06 to 1.85$)$ & 0.018 & 1.40 & (1.06 to 1.86$)$ & 0.019 & 1.28 & (0.96 to 1.71$)$ & 0.085 \\
\hline IGT & 466 & 1.62 & (1.24 to 2.10$)$ & $<0.001$ & 1.54 & (1.18 to 2.01$)$ & 0.001 & 1.49 & (1.14 to 1.95$)$ & 0.004 & 1.29 & (0.98 to 1.70$)$ & 0.070 \\
\hline Diabetes & 230 & 1.70 & (1.22 to 2.37$)$ & 0.002 & 1.64 & (1.17 to 2.30$)$ & 0.004 & 1.55 & (1.10 to 2.18$)$ & 0.013 & 1.27 & (0.89 to 1.82$)$ & 0.180 \\
\hline \multicolumn{14}{|l|}{ Elderly group } \\
\hline NFG/NGT & 1762 & 1 & & & 1 & & & 1 & & & 1 & & \\
\hline IFG & 599 & 1.23 & (1.01 to 1.49$)$ & 0.037 & 1.22 & (1.00 to 1.48$)$ & 0.049 & 1.21 & (0.99 to 1.46 ) & 0.061 & 1.13 & (0.92 to 1.37 ) & 0.236 \\
\hline IGT & 781 & 1.15 & (0.97 to 1.38$)$ & 0.116 & 1.15 & (0.96 to 1.37 ) & 0.126 & 1.16 & (0.97 to 1.39$)$ & 0.106 & 1.05 & (0.88 to 1.27 ) & 0.570 \\
\hline Diabetes & 284 & 1.12 & (0.86 to 1.45$)$ & 0.414 & 1.11 & (0.85 to 1.45$)$ & 0.425 & 1.12 & (0.86 to 1.47$)$ & 0.402 & 0.99 & (0.75 to 1.30$)$ & 0.940 \\
\hline
\end{tabular}

Model 1 included age, and sex. Model 2 additionally included eGFR, total cholesterol level, follow-up period, smoking, and drinking in model 1. Model 3 additionally included BMI in model 2.

BMI, body mass index; eGFR, estimated glomerular filtration rate; IFG, impaired fasting glucose; IGT, impaired glucose tolerance; NFG, normal fasting glucose; NGT, normal glucose tolerance.

(OR $1.49 ; 95 \%$ CI 1.14 to $1.95 ; \mathrm{p}=0.004)$, and diabetes (OR 1.55; 95\% CI 1.10 to $2.18 ; \mathrm{p}=0.013$ ) after adjusting for confounding factors including age, sex, eGFR, serum total cholesterol level, follow-up period, smoker, habitual drinker, and history of CVD (model 2). However, after taking BMI into account (model 3), the risk of hypertension in all impaired glucose metabolism categories was not statistically significantly different from those with normal glucose metabolism. In the elderly population, there were no differences in the risk of hypertension among those with NFG/NGT, IFG, IGT and diabetes.

Online supplementary table $\mathrm{S} 1$ shows insulin resistance indices in middle-aged participants between April 1990 and March 2003. HOMA-IR increased in the order of NFG/NGT, IFG, IGT, and diabetes. Matsuda index decreased in the order of NFG/NGT, IFG, IGT, and diabetes. Online supplementary figures 1A,B show that BMI was significantly associated with HOMA-IR ( $r=0.446$, $\mathrm{p}<0.001)$ and Matsuda index $(\mathrm{r}=-0.437, \mathrm{p}<0.001)$.
A BMI-stratified analysis in the middle-aged population showed that, in the non-obesity group, $144(16.1 \%$ of 897) participants with NFG/NGT, 69 (21.1\% of 327) with IFG, 77 (24.1\% of 320$)$ with IGT, and 42 (29.0\% of 145 ) with diabetes developed hypertension and that the rate of incident hypertension was significantly increased in the order of NFG/NGT, IFG, IGT, and diabetes ( $p$ for trend $<0.001)$. In the obesity group, 35 participants (28.0\% of 125$)$ with NFG/NGT, 31 (34.1\% of 91) with IFG, $42(28.8 \%$ of 146$)$ with IGT, and $19(22.4 \%$ of 85$)$ with diabetes developed hypertension. Table 3 shows the unadjusted and adjusted ORs for the incidence of hypertension in the BMI-stratified analysis in the middleaged population. In the non-obesity group, the risk of hypertension in those with IGT (OR 1.42; 95\% CI 1.03 to $1.97 ; \mathrm{p}=0.033$ ) and in those with diabetes (OR 1.77; $95 \%$ CI 1.17 to $2.69 ; p=0.007)$, but not in those with IFG, was significantly high as compared with those with NFG/ NGT. In the obesity group, there was no difference in the

Table 3 Body mass index stratified univariate and multivariate ORs for incident hypertension in middle-aged participants

\begin{tabular}{|c|c|c|c|c|c|c|c|}
\hline \multirow[b]{2}{*}{ Variables } & \multirow[b]{2}{*}{$\mathbf{N}$} & \multicolumn{3}{|c|}{ Univariate } & \multicolumn{3}{|c|}{ Multivariate } \\
\hline & & OR & $95 \% \mathrm{Cl}$ & $P$ value & OR & $95 \% \mathrm{Cl}$ & $P$ value \\
\hline \multicolumn{8}{|c|}{ BMI <25 $(n=1689)$} \\
\hline NFG/NGT & 897 & 1 & & & 1 & & \\
\hline IFG & 327 & 1.40 & (1.02 to 1.93 ) & 0.040 & 1.22 & (0.88 to 1.70$)$ & 0.231 \\
\hline IGT & 320 & 1.66 & (1.21 to 2.26 ) & 0.002 & 1.42 & (1.03 to 1.97$)$ & 0.033 \\
\hline Diabetes & 145 & 2.13 & (1.43 to 3.18 ) & $<0.001$ & 1.77 & (1.17 to 2.69 ) & 0.007 \\
\hline \multicolumn{8}{|c|}{$\mathrm{BMI} \geq 25(\mathrm{n}=447)$} \\
\hline NFG/NGT & 125 & 1 & & & 1 & & \\
\hline IFG & 91 & 1.33 & (0.74 to 2.38 ) & 0.340 & 1.25 & (0.69 to 2.28$)$ & 0.462 \\
\hline IGT & 146 & 1.04 & (0.61 to 1.76 ) & 0.889 & 0.96 & (0.56 to 1.65$)$ & 0.878 \\
\hline Diabetes & 85 & 0.74 & (0.39 to 1.41$)$ & 0.359 & 0.59 & (0.30 to 1.16$)$ & 0.129 \\
\hline
\end{tabular}

Multivariate model included age, sex, BMI, eGFR, total cholesterol level, follow-up period, smoking, and drinking.

BMI, body mass index; eGFR, estimated glomerular filtration rate; IFG, impaired fasting glucose; IGT, impaired glucose tolerance; NFG,

normal fasting glucose; NGT, normal glucose tolerance. 
Table 4 Univariate and multivariate ORs of obesity for incident hypertension in middle-aged participants

\begin{tabular}{|c|c|c|c|c|c|c|c|}
\hline \multirow[b]{2}{*}{ Variables } & \multirow[b]{2}{*}{$\mathbf{N}$} & \multicolumn{3}{|c|}{ Univariate } & \multicolumn{3}{|c|}{ Multivariate } \\
\hline & & OR & $95 \% \mathrm{Cl}$ & $P$ value & OR & $95 \% \mathrm{Cl}$ & $P$ value \\
\hline NFG/NGT non-obesity & 897 & 1 & & & 1 & & \\
\hline Obesity & 125 & 2.03 & (1.32 to 3.12 ) & 0.001 & 2.10 & (1.35 to 3.25 ) & $<0.001$ \\
\hline IFG non-obesity & 327 & 1 & & & 1 & & \\
\hline Obesity & 91 & 1.93 & (1.16 to 3.21 ) & 0.011 & 2.06 & (1.21 to 3.49 ) & 0.007 \\
\hline IGT non-obesity & 320 & 1 & & & 1 & & \\
\hline Obesity & 146 & 1.27 & (0.82 to 1.98$)$ & 0.281 & 1.19 & (0.75 to 1.87 ) & 0.456 \\
\hline Diabetes non-obesity & 145 & 1 & & & 1 & & \\
\hline Obesity & 85 & 0.71 & (0.38 to 1.32$)$ & 0.274 & 0.71 & (0.37 to 1.35$)$ & 0.301 \\
\hline
\end{tabular}

Obesity was defined as $\mathrm{BMI} \geq 25 \mathrm{~kg} / \mathrm{m}^{2}$.

Multivariate model included age, sex, eGFR, total cholesterol level, follow-up period, smoking, and drinking.

BMI, body mass index; eGFR, estimated glomerular filtration rate; IFG, impaired fasting glucose; IGT, impaired glucose tolerance; NFG,

normal fasting glucose; NGT, normal glucose tolerance.

risk of hypertension among those with NFG/NGT, IFG, IGT or diabetes.

Table 4 shows the impact of baseline obesity on the risk of hypertension in each of the baseline categories of impaired glucose metabolism in the middle-aged population. Obesity was significantly associated with an increased risk of hypertension only in those with IFG (OR 2.06; 95\% CI 1.21 to 3.49; $\mathrm{p}=0.007$ ) and those with NFG/NGT (OR 2.10; 95\% CI 1.35 to 3.25; $\mathrm{p}<0.001$ ).

Online supplementary table S2 shows the unadjusted and adjusted ORs of impaired glucose metabolism, BMI, renal dysfunction, and the presence of proteinuria on the risk of incident hypertension in participants with available urine protein levels. BMI was significantly associated with an increased risk of hypertension in both the middle-aged and elderly participants. The presence of proteinuria was significantly associated with an increased risk of hypertension in the elderly participants (OR 2.12; $95 \%$ CI 1.51 to $2.98 ; \mathrm{p}<0.001)$ but not in middle-aged participants.

\section{DISCUSSION}

In the present study, we investigated the risk of hypertension in normotensive participants with newly diagnosed different stages of impaired glucose metabolism, focusing on the difference between the middle-aged and elderly populations. In the middle-aged participants, the risk of hypertension increased in the order of NFG/ NGT, IFG, IGT, and diabetes. However, this relationship was no longer significant after adjustment for BMI. At midpoint of the study, the results showed a gradual development of insulin resistance due to impaired glucose metabolism and are strong association between BMI and insulin resistance. The confounding effect of BMI on the incidence of hypertension may be mainly due to insulin resistance. BMI-stratified analysis revealed that the risk of hypertension was higher in those with IGT and diabetes as compared with those with NFG/NGT in non-obese participants but not in obese participants.
Subgroup analysis demonstrated that obesity was significantly associated with hypertension only in those with IFG and NFG/NGT. In elderly participants, any stages of impaired glucose metabolism had no additional effects on the risk for hypertension as compared with NFG/ NGT. Conversely, proteinuria was a significant predictor of incident hypertension in elderly participants but not in middle-aged participants. Proteinuria is a marker of damage in the systemic arterial tree. ${ }^{30}{ }^{31}$ Our results suggest that the vascular injury may play a critical role in the development of hypertension in elderly participants with impaired glucose metabolism.

Several studies have reported the pronounced risk for hypertension in middle-aged diabetic populations of varying ethnicities. In the Strong Heart Study, American Indian participants with diabetes had a 2.34 times higher risk of hypertension compared with non-diabetic participants. ${ }^{6}$ Tsimihodimos et $a l^{7}$ reported around a three times higher risk of hypertension in both Hispanic whites with diabetes (in Mexico City Diabetes Study) and non-Hispanic whites with diabetes (in Framingham Offspring Study). Derakhshan $e t a l^{8}$ reported that Iranian participants with diabetes had a 2.56 times higher risk of hypertension compared with non-diabetic participants. In contrast to these previous studies, participants with diabetes had a very small risk of hypertension in this study. Our study population consisted only of participants with newly diagnosed diabetes, and no participants had a history of treatment for diabetes. This means that the diabetes group in our study was mostly comprised of individuals with the early stage of diabetes. Conversely, the diabetes group in previous studies included patients who were under the treatment with hypoglycemic medications. Patients with long-standing type 2 diabetes commonly exhibit arterial stiffness, vascular resistance, and subclinical renal dysfunction, all of which develop as duration of diabetes prolongs. ${ }^{14-16}$ These elements could be confounding factors in the relationship between type 2 diabetes and hypertension because vascular damage 
and renal dysfunction predispose to the development of hypertension. ${ }^{17} 18$ Consistent with this concept, adjustment for arterial elasticity measured by tonometry, serum cystatin $\mathrm{C}$, and urinary albumin/creatinine ratio eliminated the magnitudes of the relationship between glucose status and incident hypertension in the Multiethnic Study of Atherosclerosis. ${ }^{32}$ A recent Mendelian randomization analysis showed only a slightly increased risk of hypertension (OR: 1.06-1.09) in genetically linked type 2 diabetes, suggesting that hyperglycemia per se has only a slight impact on the incidence of hypertension. ${ }^{12}$ The pronounced impact of diabetes on incident hypertension in previous observational studies may be mainly due to confounding factors including vascular and renal damage, which were not adequately taken into account. ${ }^{13}$ Our results suggest that the risk of incident hypertension is modest in participants with the early stage of type 2 diabetes.

Regarding patients with IFG, a type of prediabetes, two studies from Japan reported that those with IFG had a higher risk of incident hypertension as compared with NGT. ${ }^{19} 33$ Conversely, those with IFG had no additional risk of incident hypertension as compared with those with NGT in both the Multi-Ethnic study of Atherosclerosis and the Strong Heart Study. ${ }^{62}$ Those with IGT, another type of prediabetes, was associated with incident hypertension in the San Antonio Heart Study. ${ }^{5}$ Conversely, Vaccaro $e t a l^{20}$ reported no significant difference in the risk of hypertension between middle-aged patients with IGT and age, sex, weight-matched groups with NGT. Two studies reported no difference in the risk of hypertension between those with IFG and IGT, but the two conclusions were the opposite. ${ }^{21}{ }^{34}$ These inconsistent results suggest the risk of incident hypertension in prediabetes is substantially lower than that in diabetes. Even in studies that showed an increased risk of hypertension in prediabetes, most reported ORs less than 2.0. ${ }^{51934}$ In this study, the increased risk of hypertension in IGT was limited to participants without obesity. IFG had no increased risk of hypertension regardless of the presence of obesity. Conversely, the presence of obesity carried nearly a twofold increased risk for hypertension in those with IFG, suggesting that obesity, but not hyperglycemia, plays a critical role in the development of hypertension in the early stages of impaired glucose metabolism. Interestingly, the presence of obesity carried no additional risk for hypertension in both those with IGT and diabetes. In terms of reducing the risk for hypertension, obesity treatment might be advantageous in the early stages rather than the advanced stages of impaired glucose metabolism.

In this study, the rate of incident hypertension was higher in participants over 65 years than under 65 years at any baseline categories of impaired glucose metabolism. However, there were no differences in the risks of hypertension among the NFG/NGT, IFG, IGT and diabetes groups in those over 65 years, suggesting that any impaired glucose metabolism had no additional effects on the risk for hypertension as compared with NFG/NGT in the elderly population. However, our results in participants with newly diagnosed diabetes might not extend to participants who are on treatment for diabetes. A recent study in the elderly population reported the difference in cardiovascular mortality between participants with newly diagnosed diabetes and those with long-standing diabetes. ${ }^{35}$ This means that elderly participants with newly diagnosed diabetes have distinct clinical features as compared with those being treated for diabetes. Further studies are warranted regarding the risk of hypertension in elderly participants with long-standing diabetes.

This study has some limitations. First, because of a retrospective cohort study design, the present findings should be confirmed by future prospective cohort studies. Second, hypertension was defined as taking antihypertensive medications and/or having systolic blood pressure $\geq 140 \mathrm{~mm} \mathrm{Hg}$ and/or diastolic blood pressure $\geq 90 \mathrm{~mm} \mathrm{Hg}$ based on a one-time blood pressure measurement, although current clinical guidelines recommend the mean value of two measurements on at least two different occasions. ${ }^{23}$ Third, there are ethnic differences in the prevalence of obesity and the pathophysiology in diabetes. ${ }^{36}$ In this study conducted in Japan, a large proportion of participants were non-obese. This may limit the application of our findings to other ethnicities. Fourth, in this study, diabetes was determined by FPG and 2-hour postload glucose during $75 \mathrm{~g}$ OGTT. We did not use hemoglobin A1C-based criteria. There are significant differences in sensitivity and specificity between the two methods. ${ }^{37}$ Our results might not extend to patients with type 2 diabetes diagnosed by hemoglobin A1Cbased criteria. Fifth, physical inactivity, unhealthy eating habits, and weight gain are known risk factors for incident hypertension. Absence of data regarding changes in lifestyle and body weight during follow-up is also study's limitation. Finally, we did not have the data on duration between the onset of diabetes and the diagnosis in the present study. However, our study population consisted only of participants with newly diagnosed impaired glucose metabolism by $75 \mathrm{~g}$ OGTT at their general health check, suggesting that the impact of diabetes duration was minimal in our study.

In conclusion, we demonstrated that the increased risk of hypertension was modest in middle-aged participants with newly diagnosed type 2 diabetes and prediabetes as compared with those with NFG/NGT. In elderly participants, no increased risk of hypertension was found in any categories of impaired glucose metabolism as compared with those with NFG/NGT. Our findings were opposite to the results of previous studies that reported the marked risk of hypertension in patients with type 2 diabetes. This discrepancy is likely due to differences in the duration of diabetes. The modest risk of hypertension in this study suggests that early stages of type 2 diabetes may be a key opportunity for reducing hypertension. In addition, our results showed that obesity was a significant predictor of the development of hypertension in those with IFG as 
well as those with NFG/NGT. Obesity might be a target for the prevention of hypertension in the early stages of impaired glucose metabolism.

Acknowledgements The authors would like to thank Naomi Yuzono for her technical and secretarial assistance.

Contributors NS conducted the analysis, interpreted data and wrote the manuscript. RO contributed to interpretation of data. RM contributed to data acquisition and interpretation of data. YH contributed to study design and interpretation of data. All authors critically reviewed and approved the final manuscript.

Funding The authors have not declared a specific grant for this research from any funding agency in the public, commercial or not-for-profit sectors.

\section{Competing interests None declared.}

Patient consent for publication Not required.

Ethics approval This study was performed in line with the principles of the Declaration of Helsinki. Ethical approval for this study was obtained from the Hiroshima Atomic Bomb Casualty Council Committee on the Ethics of Human Research (approval number 00032).

Provenance and peer review Not commissioned; externally peer reviewed.

Data availability statement Data are available on reasonable request. The datasets generated and/or analyzed during the current study are not publicly available but are available from the corresponding author on reasonable request.

Open access This is an open access article distributed in accordance with the Creative Commons Attribution Non Commercial (CC BY-NC 4.0) license, which permits others to distribute, remix, adapt, build upon this work non-commercially, and license their derivative works on different terms, provided the original work is properly cited, appropriate credit is given, any changes made indicated, and the use is non-commercial. See: http://creativecommons.org/licenses/by-nc/4.0/.

ORCID iDs

Nobuo Sasaki http://orcid.org/0000-0002-8577-7737

Yukihito Higashi http://orcid.org/0000-0001-5813-3672

\section{REFERENCES}

1 Kannel WB, Wilson PW, Zhang TJ. The epidemiology of impaired glucose tolerance and hypertension. Am Heart J 1991;121:1268-73.

2 Kahn BB. Type 2 diabetes: when insulin secretion fails to compensate for insulin resistance. Cell 1998;92:593-6.

3 Ferrannini E, Buzzigoli G, Bonadonna R, et al. Insulin resistance in essential hypertension. N Engl J Med 1987;317:350-7.

4 Higashi $\mathrm{Y}$, Oshima T, Sasaki N, et al. Relationship between insulin resistance and endothelium-dependent vascular relaxation in patients with essential hypertension. Hypertension 1997;29:280-5.

5 Haffner SM, Valdez R, Morales PA, et al. Greater effect of glycemia on incidence of hypertension in women than in men. Diabetes Care 1992:15:1277-84.

6 Wang W, Lee ET, Fabsitz RR, et al. A longitudinal study of hypertension risk factors and their relation to cardiovascular disease: the strong heart study. Hypertension 2006;47:403-9.

7 Tsimihodimos V, Gonzalez-Villalpando C, Meigs JB, et al. Hypertension and diabetes mellitus: Coprediction and time trajectories. Hypertension 2018;71:422-8.

8 Derakhshan A, Bagherzadeh-Khiabani F, Arshi B, et al. Different combinations of glucose tolerance and blood pressure status and incident diabetes, hypertension, and chronic kidney disease. J Am Heart Assoc 2016;5. doi:10.1161/JAHA.116.003917

9 Britton KA, Pradhan AD, Gaziano JM, et al. Hemoglobin A1c, body mass index, and the risk of hypertension in women. Am J Hypertens 2011;24:328-34.

10 Boyko EJ, Shaw JE, Zimmet PZ, et al. A prospective study of glycemia, body size, insulin resistance and the risk of hypertension in Mauritius. J Hypertens 2008;26:1742-9.

11 Bonnet F, Roussel R, Natali A, et al. Parental history of type 2 diabetes, TCF7L2 variant and lower insulin secretion are associated with incident hypertension. Data from the DESIR and RISC cohorts. Diabetologia 2013;56:2414-23.
12 Sun D, Zhou T, Heianza Y, et al. Type 2 diabetes and hypertension. Circ Res 2019:124:930-7.

13 Keavney B. Chicken or egg in the UK Biobank? Circ Res 2019;124:830-1.

14 Prenner SB, Chirinos JA. Arterial stiffness in diabetes mellitus. Atherosclerosis 2015;238:370-9.

15 Zoungas S, Woodward M, Li Q, et al. Impact of age, age at diagnosis and duration of diabetes on the risk of macrovascular and microvascular complications and death in type 2 diabetes. Diabetologia 2014;57:2465-74.

16 Gerstein HC, Mann JF, Pogue J, et al. Prevalence and determinants of microalbuminuria in high-risk diabetic and nondiabetic patients in the heart outcomes prevention evaluation study. The HOPE study Investigators. Diabetes Care 2000;23 Suppl 2:B35-9.

17 Safar ME. Arterial stiffness as a risk factor for clinical hypertension. Nat Rev Cardiol 2018;15:97-105.

18 Horowitz B, Miskulin D, Zager P. Epidemiology of hypertension in CKD. Adv Chronic Kidney Dis 2015;22:88-95.

19 Suematsu C, Hayashi T, Fujii S, et al. Impaired fasting glucose and the risk of hypertension in Japanese men between the 1980s and the 1990s. The Osaka health survey. Diabetes Care 1999;22:228-32.

20 Vaccaro O, Imperatore G, lovino V, et al. Does impaired glucose tolerance predict hypertension? A prospective analysis. Diabetologia 1996;39:70-6.

21 Lee CJ, Lim N-K, Kim H-C, et al. Impaired fasting glucose and impaired glucose tolerance do not predict hypertension: a community cohort study. Am J Hypertens 2015;28:493-500.

22 Suh D-C, Kim C-M, Choi I-S, et al. Comorbid conditions and glycemic control in elderly patients with type 2 diabetes mellitus, 1988 to 1994 to 1999 to 2004. J Am Geriatr Soc 2008;56:484-92.

23 Umemura S, Arima H, Arima S, et al. The Japanese Society of hypertension guidelines for the management of hypertension (JSH 2019). Hypertens Res 2019;42:1235-481.

24 American Diabetes Association. Standards of medical care in diabetes--2010. Diabetes Care 2010;33 Suppl 1:S11-61.

25 Matthews DR, Hosker JP, Rudenski AS, et al. Homeostasis model assessment: insulin resistance and beta-cell function from fasting plasma glucose and insulin concentrations in man. Diabetologia 1985;28:412-9.

26 Matsuda M, DeFronzo RA. Insulin sensitivity indices obtained from oral glucose tolerance testing: comparison with the euglycemic insulin clamp. Diabetes Care 1999;22:1462-70.

27 DeFronzo RA, Matsuda M. Reduced time points to calculate the composite index. Diabetes Care 2010;33:e93.

28 Matsuo S, Imai E, Horio M, et al. Revised equations for estimated GFR from serum creatinine in Japan. Am J Kidney Dis 2009;53:982-92.

29 Examination Committee of Criteria for 'Obesity Disease' in Japan, Japan Society for the Study of Obesity. New criteria for 'obesity disease' in Japan. Circ J 2002;66:987-92.

30 Kohara K, Tabara Y, Tachibana R, et al. Microalbuminuria and arterial stiffness in a general population: the Shimanami health promoting program (J-SHIPP) study. Hypertens Res 2004;27:471-7.

31 Hermans MMH, Henry RMA, Dekker JM, et al. Albuminuria, but not estimated glomerular filtration rate, is associated with maladaptive arterial remodeling: the Hoorn study. J Hypertens 2008;26:791-7.

32 Levin G, Kestenbaum B, Ida Chen Y-D, et al. Glucose, insulin, and incident hypertension in the multi-ethnic study of atherosclerosis. Am J Epidemiol 2010;172:1144-54.

33 Morio $\mathrm{M}$, Inoue $\mathrm{M}$, Inoue $\mathrm{K}$, et al. Impaired fasting glucose as an independent risk factor for hypertension among healthy middle-aged Japanese subjects with optimal blood pressure: the Yuport medical checkup centre retrospective cohort study. Diabetol Metab Syndr 2013:5:81.

34 Janghorbani M, Bonnet F, Amini M. Glucose and the risk of hypertension in first-degree relatives of patients with type 2 diabetes. Hypertens Res 2015;38:349-54.

35 Tang O, Matsushita K, Coresh J, et al. Mortality implications of prediabetes and diabetes in older adults. Diabetes Care 2020;43:382-8.

36 Gujral UP, Mohan V, Pradeepa R, et al. Ethnic differences in the prevalence of diabetes in underweight and normal weight individuals: the CARRS and NHANES studies. Diabetes Res Clin Pract 2018;146:34-40.

37 Sacks DB. A1C versus glucose testing: a comparison. Diabetes Care 2011;34:518-23. 